\title{
A Parallel Framework for Video Super-resolution
}

\author{
Pedro Garcia Freitas \\ Department of Computer Science, University of Brasília (UnB), Brasília, Brazil \\ Advisor: Mylène C.Q. Farias, University of Brasília (UnB), Brasília, Brazil \\ Date and location of M.Sc. defense: 19 February 2013, University of Brasília
}

Received $3^{\text {rd }}$ Feb 2014; Accepted $28^{\text {th }}$ May 2014

\section{Background}

Image super-resolution [1] algorithms are, generally, computationally expensive, since they involve a number of operations over a large data amount. When these algorithms are adapted to magnify video frames, this computational effort is further increased. Therefore, approaches that reduce the processing time of superresolution algorithms are desirable. With this aim, we propose a strategy to reduce the processing time of superresolution algorithms that uses a selective processing approach. In other words, the algorithm selects which data is to be processed exclusively by super-resolution algorithms. Combined with the selective processing technique, we also propose an approach to parallel video data processing. The combination of these two approaches allows us to build a framework to increase video resolution.

\section{Proposed Framework}

The framework consists of two major contributions. The first one we call "simplification" because it classifies the video data into complex or simple regions. Complex regions contain a higher percentage of visually significant information than simple regions. Complex regions are processed by algorithms that produce a better visual quality, which are also more computationally complex. The less expensive algorithms are used in simpler regions. As simpler regions require a smaller resource consumption, a simplification approach increases the number of these regions and decreases the number of complex regions. In this work, the proposed simplifications are significant information selection (SIS), contour-guided processing (CGP), and differential encoding (DC).

The SIS simplification strategy considers that the super-resolution algorithms are applied on the YUV color space. If the video is not encoded using YUV color space, the framework converts it to YUV and the superresolution algorithm is applied to the luminance channel. Color channels are resized using simple interpolation techniques.

The CGP strategy uses the Canny edge detector [2] to segment the regions with more or less details. The regions containing edges are classified as "regions of interest" (ROI). Then, the super-resolution algorithm is applied only on the ROI. Other regions are resized using interpolation algorithms.

Correspondence to: <sawp@sawp.com.br >

Recommended for acceptance by <Alicia Fornés and Volkmar Friken>

ELCVIA ISSN:1577-5097

Published by Computer Vision Center / Universitat Autònoma de Barcelona, Barcelona, Spain 
The DC strategy exploits the redundancy between consecutive video frames. This approach is well-known in video compression as differential coding. The redundancy elimination helps decrease the complex regions amount.

The second major contribution is the parallel processing of the super-resolution algorithm. The idea is to process, in parallel, the simplification steps. After this, the framework distributes the data and performs the super-resolution algorithm in parallel.

\section{Tests and Results}

Our tests consisted of reducing the frames of a database of videos in half and, then, increasing their resolution using the proposed framework. In our simulations, we used super-resolution algorithms with three different methods: SARTV, SARL1 [3], and SRvSC [4]. For each of these methods, we analyzed the computational performance gain using the proposed strategies. Moreover, we also investigated how the proposed strategies impacts the video visual quality.

To compute the performance gain, we measured the speedup for simplification (simplification speedup). This measure consists of calculating the ratio between the runtime using simplification and without simplification. As expected, in our tests the speedups are less for the larger block sizes. However, while smaller blocks have greater speedup, these values only illustrate the performance gain when using the simplifications. According to the speedup data, the optimum size for blocks is $32 \times 32$.

In our simulations, we observed that the smaller the block, the greater the number of regions classified as simple and, therefore, processed using interpolation. It is expected that visible degradation is inversely proportional to block size. However, we observed that the growth in quality is small for blocks larger than $32 \times 32$. Therefore, these dimensions of block size were considered a good comprise.

Besides the analysis of visual quality and simplification efficiency, we also analyzed the performance of the parallel execution. For a block size of $32 \times 32$, we varied the number of processes. From our simulations, we noticed that, in most cases, speedup increases almost linearly with the number of processes.

\section{Conclusion}

In this work, we implemented a super-resolution framework for video signals, targeted at video on demand services, which does not impact video coding strategies. A good application for this method is online hosting video, such as as Youtube, Metacafe, and Vimeo. Currently, we are investigating other ways to improve the selection criteria used by the simplification steps, which consist of replacing the contour-guided processing by a perceptual model. In addition, future studies are needed to investigate the parallel implementation of the method using different architectures (e.g. CUDA, OpenMP, Threading Building Blocks, etc.).

\section{References}

[1] R.Y. Tsai, T.S. Huang, "Multiframe image restoration and registration", Advances in computer vision and Image Processing 1(2): 317-339, 1994.

[2] J. Canny, "A Computational Approach to Edge Detection", IEEE Transactions on Pattern Analysis and Machine Intelligence 8(6):679698, 1986.

[3] S. Villena, M. Vega, S.D. Babacan, R. Molina, A. Katsaggelos, "Bayesian combination of sparse and nonsparse priors in image super resolution”, Digital Signal Processing 23(2): 530-541, 2013.

[4] J. Yang, J. Wright, T. S. Huang, Y. Ma, "Image super-resolution via sparse representation", IEEE Transactions on Image Processing 19(11): 2861-2873, 2010. 